change in the average initial proportions of the offspring and, in addition, the intensity of selection was increasing year by year.

This led Prof. Weldon to suspect that silt in the Sound, which was increasing steadily since the building of the breakwater, was the selective factor, and this he claimed to prove by laboratory experiments; crabs with relatively wide frontal apertures died, their gills covered with silt. Apparently an excellent example of effective selection in Nature, it has already been criticised on various scores by the late Mr. J. T. Cunningham ${ }^{3}$ and others.

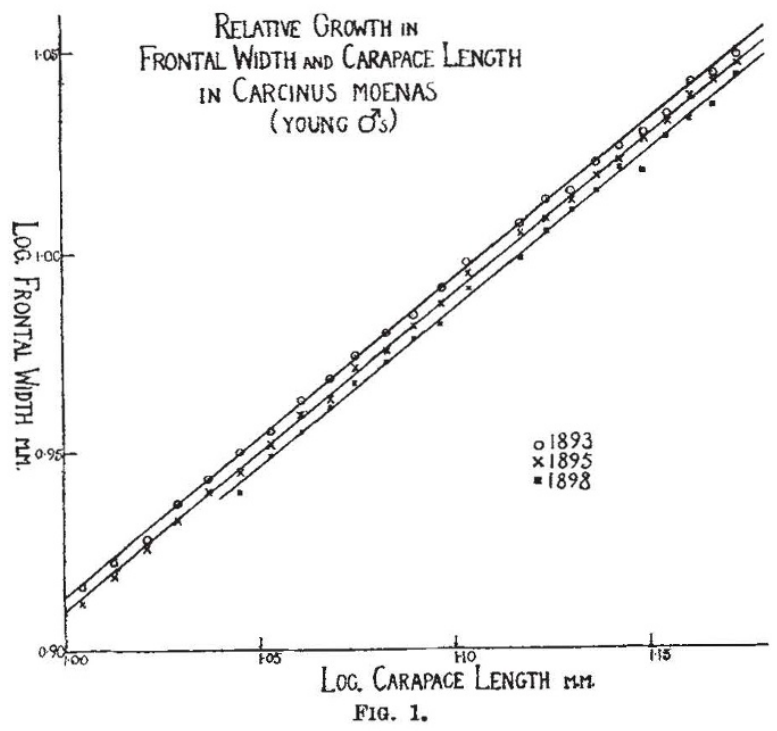

Now when the figures are plotted doublelogarithmically, frontal width against carapace length, they give a straight line indicating heterogonic growth. The change of proportions which accompanies growth therefore occurs in every individual and automatically, not merely in the group-average, as a result of natural selection. The increase, with growth, of the annual differences, is similarly automatic, given the initial differences in proportion (moasured by the constant $b$ ). The graphs for the three years are three parallel straight lines (Fig. 1), indicating identical heterogonic growth with a constant growth-coefficient, $(k=0 \cdot 8)$.

The constant $b$ is the only quantity responsible for the differences between different years. However, data are required for more than three years to show whether or not its diminution is continuing. In any event, an explanation is required of its variability. This may be the effect of a variable external condition acting on early development, or even earlier, on the egg, through the parents. Analysis on the basis of heterogony has clarified and narrowed down the problem. In conclusion, it is of interest that young females differ from young males of the same year $(1893)^{4}$, but only in the constant $b$.

Department of Zoology and

A. E. NeEDHAM.

Comparative Anatomy,

University Museum. Oxford.

1 J. S. Huxlev, "Problems of Relative Growth", London, 1932 "I. S. Huxlev, "Problems of Relative Growth", London, 193 3 W. F. R. Weinon. Section D, Brit. Ass. Rep.

"W. F. R. Weldon, Proc. Roy. Soc., 57'; 1894.

\section{Incidence of Rickets in Rabbits}

IN the Medical Research Council's Monograph "Vitamins : A Survey of Present Knowledge" (1932) it is pointed out that in Nature, rickets in rabbits is probably unknown, but can be easily produced under laboratory conditions. Furthermore, we can state that under normal conditions of management, the ordinary tame rabbit, as kept in hutches, is also free from the disorder. At this centre, about two thousand or more tame rabbits have been bred, and only three cases of rickets have been seen in the normal stock in nine years; two were in large varieties and one in a medium.

During the years 1927-29, importations of a new class of rabbit, namely, the 'Rex', with fur like the mole and which behaves as a recessive to normal fur, was imported from France and Germany, and this Institute purchased one Castorrex male and a trio of $F_{1}$ generation from Rex crossed normal coated stock, all from the same breeder. From the progeny of these animals, a number of rachitic animals appeared. Intensive breeding methods were followed in experimental fur production ${ }^{1}$. The male Rex was mated to normal coated does in May and June, 1929, and from 10 litters, $38 F_{1}$ were grown to adults, from which 9 male and 19 female $F_{1}$ were selected for interbreeding, and from February 1930 until September 1931, produced $524 \mathrm{~F}_{2}$, in addition to certain other youngsters.

Of the $F_{2}$ generation, approximately one per cent of the rex coated rabbits had badly malformed forelegs, the condition resembling severe rickets, one or both forelegs being convex (bowed), whilst others, less severely affected, had one or both forelegs concave (knock-kneed). Closer examination showed some slight abnormality in a few of the normal coated animals, but in no instance were these more than slight defects. Afterwards some adults were killed for post-mortem examination in the Veterinary Laboratory, and the severe cases confirmed as rickets, whilst a slight case was not confirmed.

Examination of the breeding records showed that rachitic animals occurred mainly in the fourth and subsequent litters, and that the disorder occurred mainly in large families. The average number of young for normal litters was $6 \cdot 3$, for litters in which concave forelegs appeared, $6 \cdot 9$, and for litters containing convex forelegs, 7.4. There was, however, one litter of one with the right foreleg pronouncedly convex. Of $99 \mathrm{~F}_{2}$ examined for abnormal forelegs there were 79 normal furred and 20 rex furred, of which 10 normal furred ( 6 were slight cases) and 3 rex coated were concave; and, 3 normal and 9 rex were convex, which is a ratio of 2.96 normal legged to 1 abnormal (rachitic). Other breeders of rex coated rabbits also reported somewhat similar cases of rickets in rex coated but not in normal coated stock. It was, therefore, decided to investigate the possible inheritance of the rachitic condition to see whether it behaved, as indicated, as a simple Mendelian recessive character. However, attempted matings of rachitic with rachitic indicated absence of sexual desire, and in no case would severely rachitic animals copulate with one another.

\section{W. KING WILSON.}

National Institute of Poultry Husbandry, Newport, Shropshire. Aug. 9.

" "Rex-Furred Rabbits", Bull. 73, Ministry of Agriculture, 1934. 